\title{
A Conceptual Framework of Space Subdivision for Indoor Navigation
}

\author{
Sisi Zlatanova \\ Liu Liu \\ GIS Technology, OTB - Research for GIS Technology, OTB-Research for \\ the Built Environment, Delft University the Built Environment, Delft University \\ of Technology \\ Jaffalaan 9, 2628 BX Delft \\ The Netherlands \\ +31(0)15278 2714 \\ S.Zlatanova@tudelft.nl
}

\author{
George Sithole \\ University of Cape Town \\ Division of Geomatics \\ Faculty of Engineering and the Built \\ Environment, Cape Town, South \\ Africa \\ +27 (0)216503576 \\ george.sithole@uct.ac.za
}

\begin{abstract}
This paper presents a conceptual foundation for the description of interiors of buildings. This framework concentrates specifically on physical and conceptual subdivisions of indoor space, which supports indoor localization and navigation. Considering user and environmental factors into account, it can be used for flexible context aware path-planning in any indoor environment. The ultimate goal of the framework is to enable automatic subdivision of indoor space and on-the-fly creation of a gridded or irregular network.
\end{abstract}

\section{Categories and Subject Descriptors}

H.1.m [Models and Principles]: Miscellaneous

\section{General Terms}

Design, Theory

\section{Keywords}

Indoor Navigation, Space Subdivision, Conceptual Framework for Subdivision Models

\section{INTRODUCTION}

Indoor navigation has recently gained a greater attention in research society and various industrial communities. Indoor navigation depends on many different technologies including indoor positioning (localisation) and tracking, indoor modelling, computation/selection of navigation paths. While much research on positioning and tracking exist, the research on models (semantic, geometric and topological) is still very fragmented and application-oriented. Models and maps are created for specific buildings, user types or applications. We argue that a unified approach is needed within indoor modelling to be able to automatically identify and derive semantics, geometry and topology of indoor space for path-finding.

There are different digital forms of virtual indoor data (both geometric and semantic). Many constructions hold their twodimensional (2D) digital floor plans in Computer Aided Design (CAD) formats. An extensive review on a large variety of possible $2 \mathrm{D}$ and some $3 \mathrm{D}$ spatial models for indoor navigation [1] shows

Permission to make digital or hard copies of all or part of this work for personal or classroom use is granted without fee provided that copies are not made or distributed for profit or commercial advantage and that copies bear this notice and the full citation on the first page. To copy otherwise, or republish, to post on servers or to redistribute to lists, requires prior specific permission and/or a fee.

ISA 13:, November 05-08 2013, Orlando, FL, USA, Copyright is held by the owner/author(s). Publication rights licensed to ACM. ACM 978-1-4503-2526-4/13/11 ..\$15.00.

http://dx.doi.org/10.1145/2533810.2533819 that most of the current methods are based on shape/geometric considerations. Semantics is specifically important for indoor navigation because it can facilitate machine processing such as identification of navigable spaces, extracting connectivity between rooms, establishing machine-readable Points of Interest (POI), and providing flexible support of guidance. Recently constructed large public buildings are designed as threedimensional (3D) Building Information Models (BIM) $[9,10]$ and contain explicit semantics of construction parts. CityGML, an Open Geospatial Consortium (OGC) standard, is another 3D data format [8] that provides semantics of indoor spaces (e.g. room, wall, etc.).

Generally those geometric/semantic models are not appropriate for indoor navigation and customized services because they have different partitioning styles of space. Those spaces might not be well-defined or closed, important semantics might be missing, and spaces could be too large or small, etc. For instance, we know that a line represents a 'wall' on 2D plan, yet we may not know which lines together compose a space. Both geometry and semantics are not sufficiently defined in those cases.

To bridge the gap between navigation requirements and current indoor digital models, we present a conceptual framework to structuralize indoor information. The resulting space subdivisions can take user and environmental factors into account and can be directly used to derive topological relationships (connectivity, accessibility).

This paper is organized as follows. Section 2 briefly discusses current partitioning methods and proposed modeling processes for indoor space. Section 3 introduces the proposed framework. Section 4 illustrates the framework's usage with an example. Section 5 concludes this paper with future work.

\section{NAVIGATION SPACES}

The research of indoor modelling is originated from two domains namely robot navigation and human navigation. While navigation approaches in robotics are based on regular (grid) subdivision of space, human navigation is mainly built upon irregular networks. Besides, human navigation makes extensive use of semantics. Reviews of existing navigation models and approaches for path finding can be found in many articles $[1,7,24]$.

The navigation approaches can be analysed with respect to different criteria: type of the space-subdivision - regular (e.g. grid) or irregular (network); geometric dimension of the used models such as 2D, 2,5D (surface), 3D; and semantic specification of the 
space such as geometric, topological, semantic models or combinations of them. In our framework, a very important criterion is the semantic identification of space.

Looking at semantics, we can see different trends in use of semantics. A large group of the current approaches are geometry (topology)-based with no or little use of semantics. Typically these are based on regular grids such as rectangular, hexagon, octagon, etc. in 2D or 3D space $[2,6,11,13,25]$. Topology is derived by eg applying K-nearest neighbour algorithms.

Another group of approaches concentrates on irregular subdivision of space from which a network is derived $[3,4,12$, $14,16,17,21,22,23]$. In contrast to the previous group, semantics is frequently used here to identify the connectivity or accessibility between indoor sub-spaces. For example, the notions of 'door' and 'window' are used to derive the connection between two 'rooms'.

Third group of approaches concentrate heavily on semantics by building hierarchical or multi-layered conceptualisations of indoor environments $[3,15,18,19,20]$. These hierarchies are created by aggregation or specialisation of building units according to different parameters, such as structural division (physical closing), functional use (e.g. offices, passages), organization/affiliation, etc. $[3,18,19]$. The subdivisions could be even independent from the building structure, e.g. sensor coverage area [3].

Semantics concepts broaden the indoor navigation problem from pure path-finding in an enclosed space to the more elaborate problem of multi-user, multi-purpose navigation in changing complex environments. In order to provide such a flexible navigation style, knowledge about indoor space, users and the factors that change the environment have to be considered. Depending on the users and/or the dynamic factors space subdivision may change.

Current navigation approaches consider these components in isolation or they are only loosely coupled. Most commonly the space subdivision is created for one (or limited) group(s) of users yet functional modifications of indoor space are not taken into consideration.

Recently, several publications draw the attention to flexible modelling of indoor environments with respect to users, navigation tasks, possible influences of changing factors (e.g. crowdedness, fire, temporal renovation), i.e. to providing a context-aware navigation $[1,3,5,15]$. For example, [1] distinguishes between 'context of use' and 'context of execution'. 'Context of use' refers to Person, Location, Action. 'Context of execution' refers to Infrastructure and Performance. The MultiLayered Space Event Model (MLSEM) is another example of a user-based and event-based space subdivision [3]. But in MLSEM no explicit notations are given for users or indoor environments.

The framework presented in this paper establishes explicit relations between users (and their characteristics), their activities and dynamically changing environments. The ultimate goal is automating subdivision of indoor space and on-the-fly creating gridded or irregular network that would serve any context.

\section{CONCEPTUAL FRAMEWORK}

All concepts of this framework are illustrated with the help of the 3D ground-floor model of "Eye Medikal Hospital" uploaded by user "Vasfi Bicer" on Dec.10, 2008 at SketchUp 3D Warehouse.

\subsection{Elements of the Framework}

The framework is based on six general concepts: Space, Partition, Agent, Activity, Resource and Modifier.
Space: it is defined broadly as the environment in which humans store resources (items of interest) and engage in navigation activities. For example, the hospital is a space in which navigation tasks associated with seeing doctor can be conducted (Figure 1). A logical compartmentalisation of resources and navigation activities requires the creation of sub-spaces.

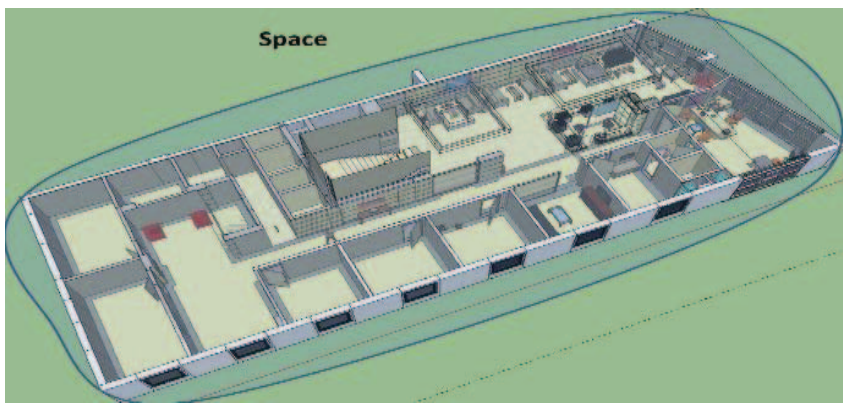

Figure 1. An example of indoor space.

Sub-spaces: are the unit spaces within indoor space and they possess properties and fulfil functions. Normally sub-spaces can be living space, work space, leisure space, storage rooms, passage, etc. Figure 2 indicates two types of sub-spaces, namely free and inert sub-spaces.

Free sub-spaces: they are units accessible by agents. Agents are navigated in free sub-spaces.

Inert sub-spaces: they are spaces in which no navigation activity can ever take place. They can be physical boundary of free subspaces. Walls and obstacles are common instances of inert subspaces.

Sub-Spaces

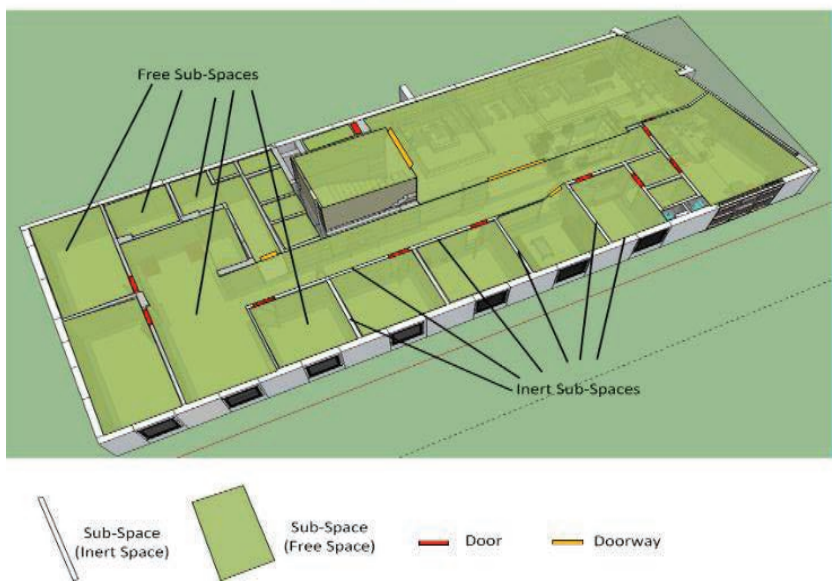

Figure 2. Illustration of sub-spaces

Partition: it is a decomposition/subdivision procedure on a space. Sub-spaces are derived in the procedure. The partition can be determined by design requirements, physical restrictions or by spatial cognition of agents.

A partition can be constructed in hierarchy of semantically defined sub-spaces. Hierarchical representation is indicated by the fact that a space consists of sub-spaces with different granularity.

The creation of sub-spaces discretises a space. However, subspaces themselves are still continuous. For instance, in Figure 2 a room/corridor can be a free sub-space. A door's panel itself is an inert sub-space (which cannot be entered in), while the sub-space 
occupied by the door panel is free. A solid wall (with thickness) is an inert sub-space as well. In short, all of sub-spaces collectively compose the space, that is, the indoor environment.

Agents: they are clients that engage in certain navigation tasks or use resources located in sub-spaces. Typically agents will be humans, but they can be human proxies as well. For instance, an agent can be a pedestrian, a robot or a remote-controlled vehicle. The general procedure for defining a proxy's sub-spaces is the same to that for human users, therefore human and proxies can be treated as indistinguishable. Agents can also from time to time act as resources within a sub-space. For example, nurses and doctors are resources for patients.

Activities: they are the tasks and navigation behaviour that an agent can perform within sub-spaces. Mostly activities are linked to navigation tasks, such as a serial of movements (under guidance) between different cites with some actions (e.g. checking in). Generally navigation task is about how an agent utilizes recourses to operate his/her planed behaviour in those sub-spaces.

Resources: they are the things that an agent can use in a sub-space or take from a sub-space. During navigation resources influence the behavior of agents. This influence (attraction or repulsion) can be specific to certain agents and its scope can be local or global. For example, a visitor to hospital may be drawn to the gift shop even though his/her goal is visiting a patient.

Modifiers: they indicate the final result generated by a certain event (e.g. moving furniture, temporary closing, fire, gas leak, blocked corridors, etc.). A modifier promotes or limits the use of a sub-space. Modifiers influence paths in a navigation action by extension of the partition of a space. Furthermore, modifiers define what event impacts which agents on which aspects. Thus modifiers may distinctly influence different agents.

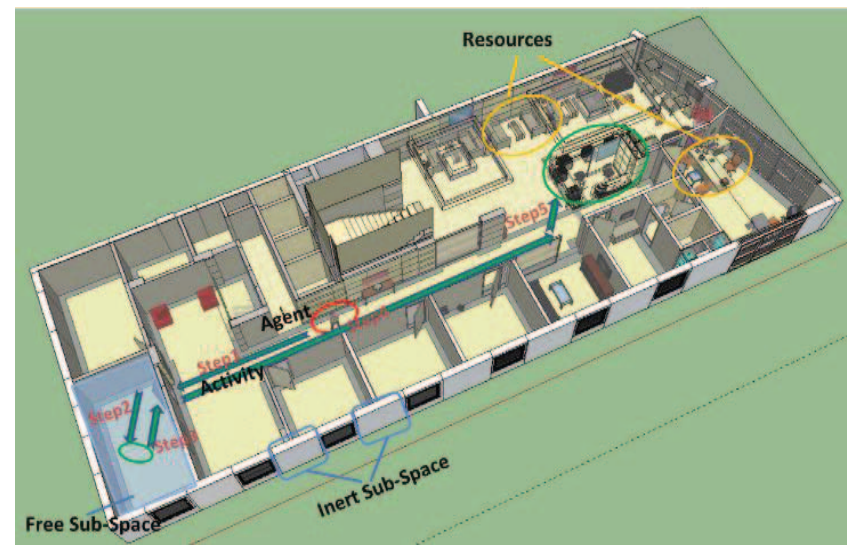

Figure 3. Agent and navigation task

Figure 3 illustrates the elements of space. An agent, a person, is bounded by a red ellipse. Yellow ellipses denote the indoor objects (chairs, sofa, desks, etc.) regarded as resources to this agent. The green lines with arrows represent the activity of the agent. The entire activity includes 1) heading to a doctor's office, 2) entering into the office and receiving diagnosis, 3) leaving the office, 4) going along with the corridor, and 5) heading to reception.

Generally, indoor navigation is based on partition of a space (grids or network). The partition (denoted by $P$ ) can be seen as a function of other elements of the framework. That is $P(A g, R, A c$, $M$ ) where $A g, R A c$ and $M$ are agent(s), resource(s), activities and
Modifier(s) respectively. As each (type of) agent(s) corresponds to an independent partition, this framework is a kind of extension of MLSEM, which means partition for one (type of) agent(s) is one Layer of the MLSEM.

The function $P(A g, R, A c, M)$ contains temporal property. When changes/events happen, the influence will be input the function via Modifier(s). It can be a succession of the change of partition: the next partition results from the previous one. Moreover, resource(s), activities can be variable along elapsed time.

\subsection{Criteria of Partition}

A space can be separated into different sub-spaces via partition in terms of different criteria including physical, conceptual and functional ones.

Figure 4 shows two different partitions in the same space. In Figure $4 \mathrm{a}$, there are two sub-spaces which are bounded by walls and enclosed by some virtual surfaces (e.g. doorway). This case involves both physical and conceptual constraints.

Figure $4 \mathrm{~b}$ gives six sub-spaces in the same space, and four of them are the units containing resources. The other two sub-spaces are free sub-spaces which are the first choice for agent's "movement" activity. Here the partition concerns functional criterion.

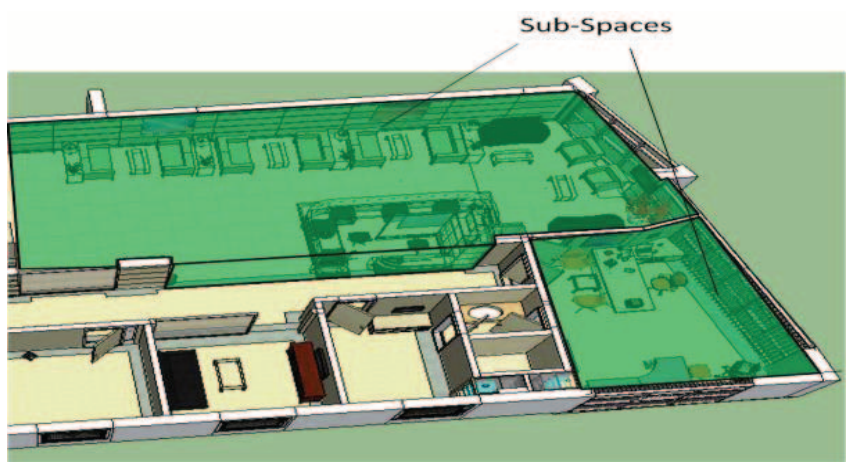

(a)

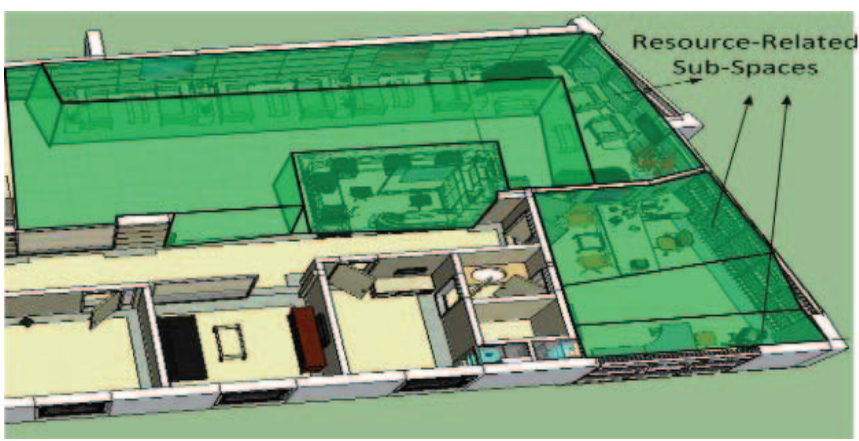

(b)

Figure 4. (a) Sub-spaces separated by walls; (b)Resourcerelated sub-spaces of the space

\subsection{Navigation Usage}

Figure 5 illustrates how a navigation process with the framework looks like. We begin with the representation of an indoor space. According to a request, partition is promoted for decomposition of the space and Modifiers have to be determined. Then subspaces are generated based on the needs of agent(s). Within subspaces the activities of agents is planned. Resources are not only considered for partition of the space, but also utilized for 
navigation in those sub-spaces. A complete path-planning is done by using all the framework elements together.

In order to automate this navigation computation, digital data should be structured, formalized and used under this framework. For instance, if we know different slope values of floor surfaces in sub-spaces, we can organize agents' demands on path-flatness. In addition, fire prevention equipment is seen as a resource. Hence the area containing such equipment can be highlighted for possible partitions. Another example is summarizing events and estimating their effects. This information should be denoted in modifiers.

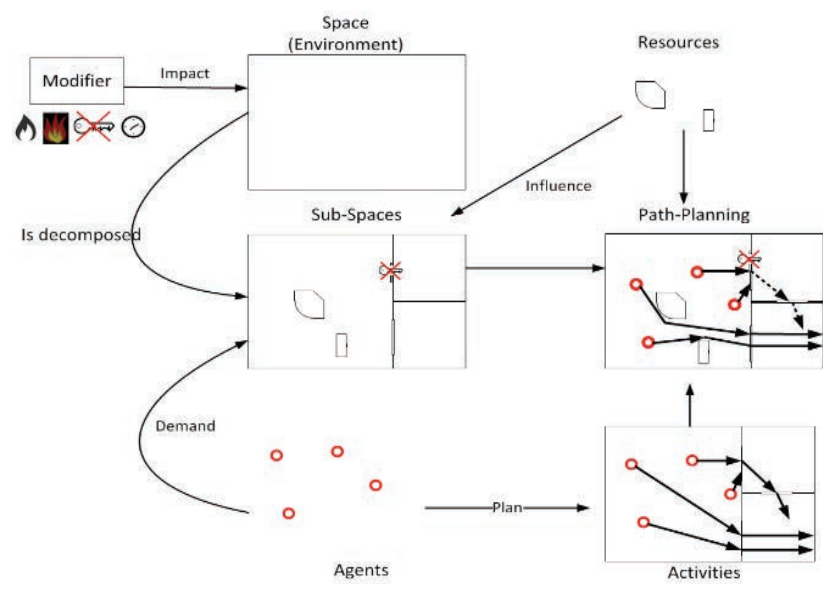

Figure 5. Illustration of navigation supported by the framework.

\section{EXAMPLE}

The framework presented in the previous section is illustrated with another example to conceptually discuss the envisioned results. Since this paper focuses on the space-decomposition topic, this section only exemplifies the effect of partition of a space.

Figure 6 denotes partitions varied with respect to modifiers. At the initial state all the free sub-spaces are green. Supposing two sites catch fire (modifier), then a new decomposition of the space is executed. The sub-spaces near heat sources are denoted with a blue color. The relative safe areas are still in green color. As smoke (modifier) diffuses along with the fire, due to the limited safe sub-spaces another partition is done. In the bottom-right area, units containing resources are separated from others. Then the only free sub-space among them is used for movement.

From the bottom decomposition in Figure 6 we can see that the green has shrunk and there are more blue sub-spaces imperilled by fire and smoke. In the example it is apparent that the partitions don't completely follow physical constraints. Different conceptual and functional shapes of sub-spaces can be taken into account.

\section{CONCLUSIONS}

According to the modelling methods for I-space and navigation, space definition, subdivision and aggregations are important aspects of indoor navigation. Space has been geometrically subdivided to provide better paths for indoor navigation. Meanwhile, semantics descriptions and properties have been increasingly introduced.

Semantics is becoming more important because the complexity of spaces and the tasks of users increase. The semantics is intended to qualitatively enhance the computed path. By using semantic annotations indoor routing can be better adapted to locomotion modes and the tasks of users.

Theoretically, the proposed framework can fulfill the user requests on indoor navigation as specified in [5]. The core is partitions which would provide different sub-spaces and related resources to certain agents. Moreover, modifiers bring real-time changes in partitions. Partitions are determined by the other elements of the framework.
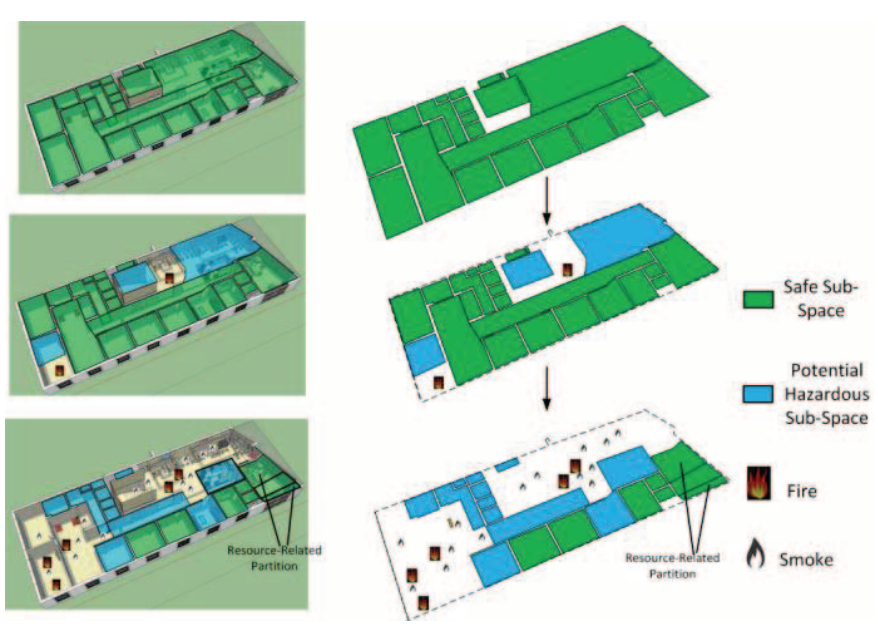

Figure 6. A serial of changes of the partition

Based on these decompositions (partitions), a graph structure can readily be constructed for path-finding. Then available paths can be computed for current agent(s).

Specifically, logical and metric graphs of indoor space can be derived from the framework with partitions. Modifiers can store and provide changing information. Moreover, the framework can offer customized decomposition.

The next step is developing the logical framework: Agents, recourses, activities and modifiers have to be developed in details. Parallel to this we need elaborate on data modelling and implementation methodology. Then we will test this framework to compare it with different navigation models, and prove the framework can manipulate those models' elements together for various navigation requests.

\section{ACKNOWLEDGMENTS}

This research was supported by a grant (11 High-tech G11) from Architecture \& Urban Development Research Program funded by Ministry of Land, Infrastructure and Transport of Korean government. We are grateful to our colleagues Junqiao (John) Zhao, Zhizong Wang and Fillippo Mortari for their useful comments and contributions at earlier stages of the paper.

\section{REFERENCES}

[1] Afyouni, I., C. Ray, and C. Claramunt, 2012, Spatial models for context-aware indoor navigation systems: A survey, Journal of Spatial Information Science, Number 4 (2012), pp. 85-123

[2] Bandi, S. \& Thalmann, D. 1998. Space discretization for efficient human navigation. Computer Graphics Forum 17(3): 195-206.

[3] Becker, T., C. Nagel, T.H. Kolbe, 2008, A Multilayered Space-Event Model for Navigation in Indoor Spaces. In: Lee, 
Zlatanova (eds.). 3D Geo-Information Sciences, Lecture Notes in Geoinformation and Cartography, 2009, Part II, 6177.

[4] Boguslawski, P. and Gold, C., 2010, Construction Operators for Modelling 3D Objects and Dual Navigation Structures, in Jiyeong Lee and Sisi Zlatanova (eds.), $3 D$ Geo-Information Sciences (Lecture Notes in Geoinformation and Cartography; Springer Berlin Heidelberg), pp. 47-59.

[5] Brown, G., C. Nagel, S. Zlatanova and T.H. Kolbe, 2013, Modelling 3D Topographic Space Against Indoor Navigation Requirements, In J. Pouliot, S. Daniel, F. Hubert and A. Zamyadi (Eds.), Progress and New Trends in $3 D$ Geoinformation Science, LNG\&C, Springer, Heidelberg, New York, Dordrecht, London, pp. 1-22

[6] Girard, G., S. Côté, S. Zlatanova, Y. Barette, J. St-Pierre and P. van Oosterom, 2011, Indoor Pedestrian Navigation Using Foot-Mounted IMU and Portable Ultrasound Range Sensors, In: Sensors 2011, Volume 11, pp. 7606-7624

[7] Giudice, N.A., Walton, L.A., \& Worboys, M. The informatics of indoor and outdoor space: A research agenda. Second ACM SIGSPATIAL International Workshop on Indoor Spatial Awareness (ISA 2010), November, San Jose, CA. 2010.

[8] Gröger, G., Kolbe, T. H., Nagel, C., and Häfele, K. 2012. OpenGIS City Geography Markup Language (CityGML) Encoding Standard v2.0.0. Open Geospatial Consortium Standard. Open Geospatial Consortium.

[9] Isikdag, U and S. Zlatanova, 2009, A SWOT analysis on the implementation of BIM within geospatial environment, in Krek, Rumor, Zlatanova \& Fendel (eds.), In: Urban and Regional data Management, UDMS Annuals 2009, 15-30 Boca Raton: CRC press, Taylor \& Francis

[10] ISO 16739:2013 (2013).Industry Foundation Classes (IFC) for data sharing in the construction and facility management industries.

[11] Kuffner, J., 1998, Goal-directed navigation for animated characters using real-time path planning and control, Lecture Notes in Computer Science: 171-186

[12] Lee, J. 2004. A Spatial Access-Oriented Implementation of a 3-D GIS Topological Data Model for Urban Entities. Geoinformatica 8(3): 237-264.

[13] Li, X., C. Claramuntb and C. Rayb, 2010, A grid graphbased model for the analysis of 2D indoor spaces. Computers, Environment and Urban Systems, Vol. 34 (6), pp. 532-540.

[14] Liu, L. and S. Zlatanova, 2011, A 'door-to-door' path-finding approach for indoor navigation, In: Antalya, Backhause,
Boccardo\&Zlatanova (Eds.), International Archives ISPRS XXXVIII, 7th Gi4DM, 3-7 May, Anltalya, Turkey, 6p.

[15] Liu, L. and S. Zlatanova, 2012, A semantic data model for indoor navigation. In P Kröger, E Tanin \& P Widmayer (Eds.), ISA '12 Workshop papers (pp. 1-8). s.1.: SIGSPATIAL

[16] Lorenz, B. Ohlbach, H.J. \& Stoffel, E.P. 2006. A Hybrid Spatial Model for Representing Indoor Environments. In Proceedings of W2GIS (LNCS 4295): 102-112. Hong Kong, China.

[17] Meijers, M. Zlatanova, S. \& Preifer, N. 2005. 3D geoinformation indoors: structuring for evacuation. In Proceedings of Next generation 3D city models. Bonn, Germany

[18] Richter, K.-F.; Winter, S.; Rüetschi, U.-J. (2009): Constructing Hierarchical Representations of Indoor Spaces. In: Tseng, Y.-C.; Scheuermann, P.; Güting, R. H. (Eds.), Tenth International Conference in Mobile Data Management, Workshop on Indoor Spatial Awareness. IEEE Press, Taipei, Taiwan, pp. 686-691.

[19] Richter, K.-F.; Winter, S. (2011): Hierarchical Representation of Indoor Spaces. Environment and Planning B, 38 (6): 1052-1070.

[20] Rüetschi, U.-J. (2007): Wayfinding in Scene Space Modelling Transfers in Public Transport. PhD thesis, Mathematisch-naturwissenschaftliche Fakultät, University of Zurich, Zurich, Switzerland, 134 pp.

[21] Schaap, J. 2010. Towards a 3D geo-data model to support pedestrian routing in multimodal public transport travel advices. MSc Thesis. GIMA MSc. programme, Utrecht University, Netherlands.

[22] Slingsby, A. \& Raper, J. 2008. Navigable Space in 3D City Models for Pedestrians. In Van Oosterom P. Zlatanova, S. Penninga, F. \& Fendel, E. (eds), Advances in $3 D$ Geoinformation Systems: 49-64. Berlin: Springer

[23] Thill, J.-C., T.H.D. Dao and Y. Zhou, 2011, Travelling in the three-dimecional city: applications in route planning, accessibility assessment. Location analysis and beyond, Journal of Transport Geography, 19 (12011), pp. 405-421

[24] Worboys, M.F., Modeling indoor space (keynote). Third ACM SIGSPATIAL International Workshop on Indoor Spatial Awareness (ISA 2011), November, Chicago, IL. 2011.

[25] Yuan, W. \& Schneide, M. 2010. Supporting 3D Route Planning in Indoor Space Based on the LEGO Representation. In proceedings of ISA'10. New York: ACM. 